\title{
OPEN Alteration of serum amino acid profiles by dietary adenine supplementation inhibits fatty liver development in rats
}

\author{
Hiroki Nishi ${ }^{1,3}{ }^{3}$ Daisuke Yamanaka ${ }^{2,3}$, Masato Masuda ${ }^{1,3}$, Yuki Goda ${ }^{1}$, Koichi Ito ${ }^{2}$, \\ Fumihiko Hakuno $^{1 \bowtie}$ \& Shin-Ichiro Takahashi ${ }^{1}$
}

Studies on animal models have demonstrated that feeding a low-arginine diet inhibits triacylglycerol (TAG) secretion from the liver, resulting in marked fatty liver development in rats. Here, we first showed that culturing hepatocytes in the medium mimicking the serum amino acid profile of lowarginine diet-fed rats induced TAG accumulation in the cells, indicating that the specific amino acid profile caused TAG accumulation in hepatocytes. Dietary adenine supplementation completely recovered hepatic TAG secretion and abolished hepatic TAG accumulation in rats. A comprehensive non-linear analysis revealed that inhibition of hepatic TAG accumulation by dietary adenine supplementation could be predicted using only serum amino acid concentration data. Comparison of serum amino acid concentrations indicated that histidine, methionine, and branched-chain amino acid (BCAA) concentrations were altered by adenine supplementation. Furthermore, when the serum amino acid profiles of low-arginine diet-fed rats were altered by modifying methionine or BCAA concentrations in their diets, their hepatic TAG accumulation was abolished. Altogether, these results suggest that an increase in methionine and BCAA levels in the serum in response to dietary arginine deficiency is a key causative factor for hepatic TAG accumulation, and dietary adenine supplementation could disrupt this phenomenon by altering serum amino acid profiles.

Nutritional status of macronutrients significantly affects systemic metabolism of animals, and their imbalanced intake leads to a series of metabolic diseases such as non-alcoholic fatty liver disease (NAFLD). NAFLD is defined as a series of hepatic diseases resulting from hepatic steatosis that develops without apparent excessive alcohol consumption. Among individuals with NAFLD, many remain under benign conditions (non-alcoholic fatty liver [NAFL]), whereas others exhibit non-alcoholic steatohepatitis (NASH) which can progress to liver inflammation, cirrhosis, and ultimately hepatocellular carcinoma ${ }^{1,2}$. Thus, an epidemic of NAFLD and NASH has increasingly given rise to clinical concerns worldwide.

For decades, the dietary imbalance of not only lipids or sugars but also other nutrients, including proteins/ amino acids and nucleobases, has been reported to have significant impacts on neutral lipid accumulation in the liver, both in rodent models and in humans ${ }^{2-5}$. Because the detailed molecular mechanisms of NAFL progression have not yet been elucidated, there is no established definitive treatment for NAFLD; but dietary supplementation with some nutrients or small compounds is currently recommended ${ }^{3}$. For example, the purine nitrogenous base adenine can completely inhibit hepatic triacylglycerol (TAG) accumulation induced by a low-arginine diet, and the pyrimidine derivative orotic acid promotes fatty liver in rats ${ }^{6,7}$. Since it is known that dietary arginine deficiency alters nucleotide metabolism in the liver, thereby increasing orotic acid biosynthesis ${ }^{8,9}$, some researchers have raised the possibility that fatty liver induced by a low-arginine diet may be caused by increased orotic $\operatorname{acid}^{10}$. However, in this case too, the detailed molecular mechanisms still remain unclear as to how modified nucleotide metabolism or the related metabolites alter the hepatic lipid metabolism.

Many factors such as obesity, genetic background, viral infections, and dietary habits have been associated with NAFLD progression, but most of its underlying mechanisms and biomarkers for diagnosis have not been defined due to its high complexity , $3,11,12$. A previous study demonstrated that a low-protein/amino acid diet and

${ }^{1}$ Department of Animal Sciences and Applied Biological Chemistry, Graduate School of Agriculture and Life Sciences, The University of Tokyo, 1-1-1 Yayoi, Bunkyo-ku, Tokyo, Japan. ${ }^{2}$ Department of Veterinary Medical Sciences, Graduate School of Agriculture and Life Sciences, The University of Tokyo, Tokyo, Japan. ${ }^{3}$ These authors contributed equally: Hiroki Nishi, Daisuke Yamanaka and Masato Masuda. ${ }^{\boxplus}$ email: hakuno@g.ecc.u-tokyo.ac.jp 
a low-arginine diet resulted in significant fatty liver development ${ }^{4,5}$, which led to the assumption that dietary arginine intake could have an important role in NAFLD progression. However, dietary arginine intake did not correlate with hepatic TAG levels, and a low-amino acid diet and a low-arginine diet induced fatty liver through a different mechanism, although both diets contained the same amounts of arginine ${ }^{13}$. This suggests that dietary amino acid concentrations are not primarily important; instead, serum amino acids are associated with hepatic lipid metabolism. In a previous study, a mathematical non-linear analysis based on a machine learning method revealed that a comprehensive serum amino acid profile, but not individual amino acid concentrations, correlated well with hepatic TAG levels in a rat model $^{5}$.

Currently, many reports have suggested that serum amino acid profiles can be used as clinical diagnostic markers, since there is evidence for serum concentrations of some amino acids becoming up- or down-regulated in particular physiological disorders such as obesity, diabetes mellitus, some cancer types, and NAFLD ${ }^{14-17}$. We also demonstrated the correlation between serum amino acid profiles and hepatic TAG levels ${ }^{5}$. These results indicate that there is a strong link between serum amino acid profiles and hepatic lipid metabolism. However, since the available reports have used correlation analyses, we cannot determine whether the change in the serum amino acid profile is the cause or consequence of the disorder in question. Therefore, in the present study, we aimed to evaluate the possible role of serum amino acids in hepatic lipid accumulation by using our original methods: a cell-culture model and a non-linear machine leaning analysis. Furthermore, we illustrated how nucleobases are associated with serum amino acid profiles and affect lipid accumulation in the liver.

\section{Results}

A specific serum amino acid profile causes TAG accumulation in hepatocytes in a cell-autonomous manner. As previously reported ${ }^{5}$, a low-arginine $(\Delta \mathrm{Arg})$ diet significantly induced TAG accumulation in the livers of rats in seven days, and serum amino acid profiles correlated well with liver TAG levels. Therefore, to evaluate the roles of serum amino acids in liver TAG accumulation, we compared the serum amino acid concentrations between $\Delta \mathrm{Arg}$ diet-fed rats and those fed a control diet. According to our results, the $\Delta \mathrm{Arg}$ diet increased or tended to increase the concentrations of four amino acids (methionine, histidine, glutamine, and glutamic acid), and reduced the concentrations of seven amino acids (cysteine/cystine, tryptophan, phenylalanine, tyrosine, arginine, threonine, and glycine), when compared to a control diet (Fig. 1a).

Next, we examined whether this specific serum amino acid profile in response to a $\triangle$ Arg diet triggered TAG accumulation in the liver. For this, a culture medium that mimicked the serum composition of $\Delta$ Arg diet-fed rats was prepared (see Supplementary Table S1). To this $\Delta$ Arg-mimic medium, twice as much of the four upregulated amino acids mentioned above as the control medium was added, and the seven down-regulated amino acids were not added. H4IIE rat hepatoma cells were cultured in control or $\Delta$ Arg-mimic medium for $24 \mathrm{~h}$, and the total TAG content was measured. The results demonstrated that the $\Delta$ Arg-mimic medium significantly enhanced TAG accumulation in the cells when compared to the control medium (Fig. 1b), indicating that the specific amino acid profile of the hepatocytes' surrounding environment could directly cause TAG accumulation. This also indicated that TAG accumulated in a cell-autonomous manner and under serum-free conditions.

Adenine supplementation restores TAG secretion in the liver of $\Delta$ Arg diet-fed rats. It has been reported that adenine supplementation in a $\triangle A$ rg diet completely abolishes TAG accumulation in the liver (Fig. 1c, Supplementary Table S2) ${ }^{6}$. Therefore, we tested the effects of adenine and purine metabolites on hepatic TAG accumulation in a rat model. Interestingly, among purine nitrogenous bases (adenine, guanine) and adenine-related metabolites (adenosine, adenosine monophosphate [AMP], and inosine monophosphate [IMP]), only adenine supplementation inhibited hepatic TAG accumulation (Fig. 1c,d, Supplementary Table S3). While a $\triangle$ Arg diet inhibited hepatic TAG secretion, resulting in fatty liver $^{13}$, our results indicated that dietary adenine, but not guanine, fully rescued TAG secretion in the liver (Fig. 1e, f).

Hepatic purine metabolism cannot explain the mechanism of fatty liver prevention by dietary adenine supplementation. To evaluate the effects of dietary arginine deficiency and the supplementation of adenine and related molecules on hepatic purine metabolism, liver samples from rats in Fig. 1d were used in the analysis of related metabolites (Fig. 2a). Interestingly, supplementation with a certain compound did not necessarily increase the concentration of its corresponding metabolite in the liver, and hepatic adenine levels did not correlate with hepatic TAG levels, suggesting that hepatic adenine per se was not very significant for fatty liver progression. In contrast, dietary adenine supplementation increased hepatic IMP levels, which negatively correlated with liver TAG levels, while no other tested metabolite displayed any correlation (Fig. 2a).

In light of these results, we evaluated rats fed a diet containing three times as much IMP or those received IMP injection directly into the tail vein. The strong dietary IMP supplementation only slightly decreased $\Delta$ Arg diet-induced hepatic TAG accumulation, while the direct intravenous injection did not show any significant effects on hepatic TAG levels (Fig. 2b,c). Collectively, these results indicate that the inhibition of $\Delta$ Arg dietinduced fatty liver caused by dietary adenine occurs through a mechanism that cannot be explained by changes in purine metabolite levels in the liver.

Multi-layer perceptron (MLP) analysis can predict the suppression of TAG accumulation only using serum amino acid profiles. In an earlier study from our group, we evaluated 135 data sets with serum amino acid concentrations and hepatic TAG levels in rats fed 27 different diets with varying amino acid compositions. A machine learning program based on MLP was established to predict individual hepatic TAG levels using the data on serum amino acid concentrations ${ }^{5}$. Based on this method, serum amino acid profiles and hepatic TAG levels were shown to exhibit a good correlation. Therefore, we analyzed the serum amino acid 
(a)
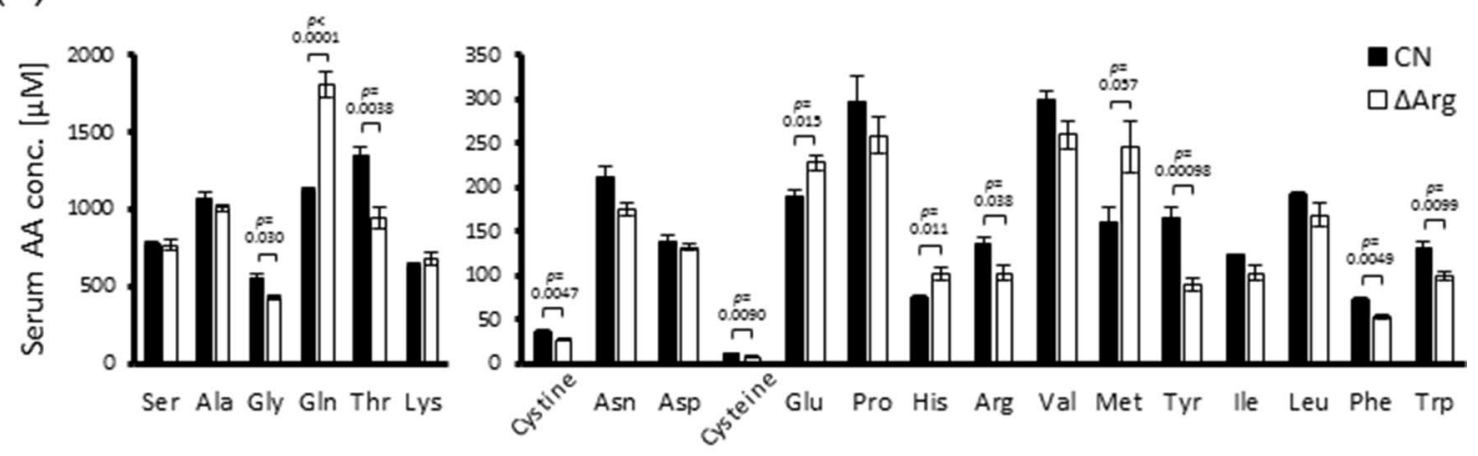

(b)

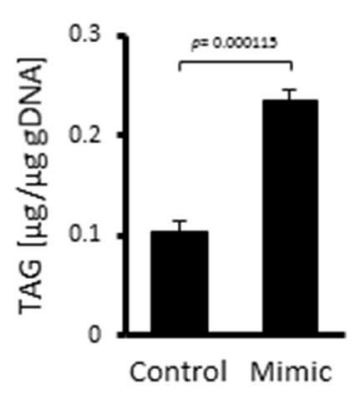

(c)

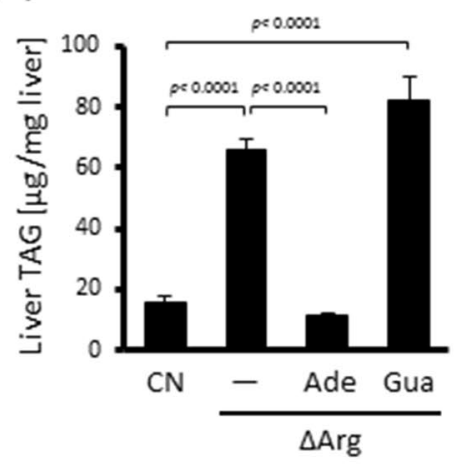

(d)

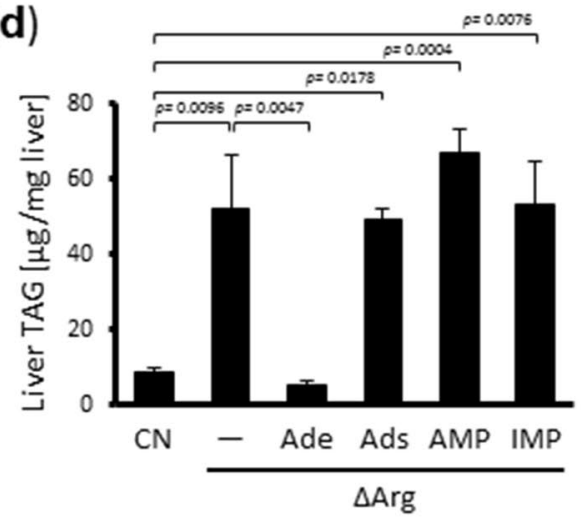

(e)

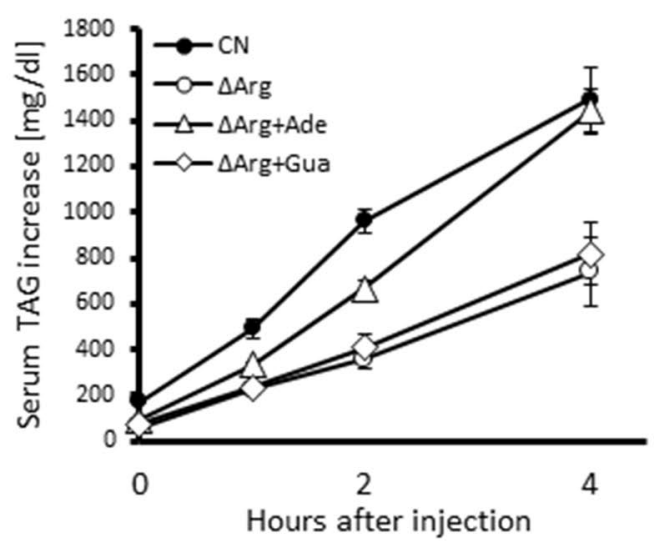

(f)

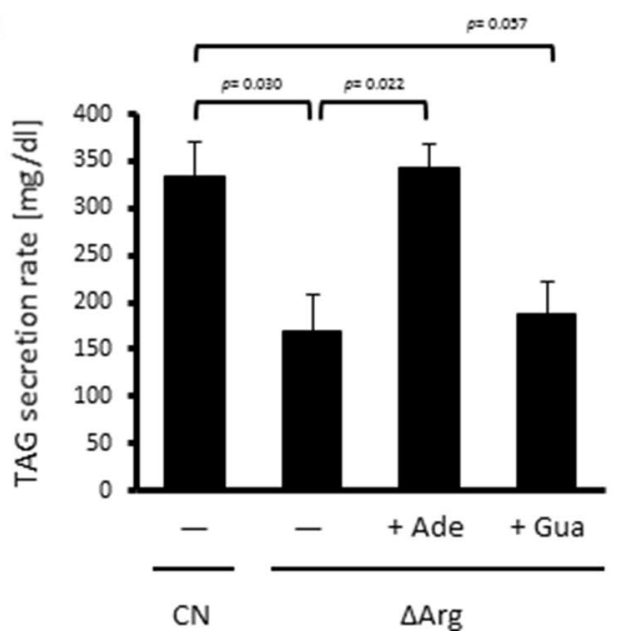

Figure 1. Dietary adenine supplementation reverses arginine deficient diet-induced lipid accumulation in the liver. (a) Six-week-old male Wistar rats were fed a control amino acid-mixed diet (CN) or low-arginine diet $(\triangle \mathrm{Arg})$ for seven days, then bloods from the carotid artery were collected following $1 \mathrm{~h}$ fast. Sera were prepared and their amino acid concentrations were measured by LC-MS/MS. (b) H4IIE rat hepatoma cells were cultured for $24 \mathrm{~h}$ in a control medium or a medium mimicking serum amino acid composition of $\Delta$ Arg diet-fed rats. TAG levels in the cell were measured. (c, d) Six-week-old male Wistar rats were fed the CN diet or $\Delta$ Arg diet with or without adenine (Ade), guanine (Gua) (c), adenosine (Ads), adenosine-monophosphate (AMP) or inosine-monophosphate (IMP) (d) supplementation at the concentration of $3 \mathrm{~g} / \mathrm{kg}$ for seven days. TAG levels in their livers were measured. (e, f) Six-week-old male Wistar rats were fed a CN diet or $\triangle$ Arg diet with or without $3 \mathrm{~g} / \mathrm{kg}$ adenine or guanine supplementation for seven days. After $16 \mathrm{~h}$ fasting on the last day, tyloxapol was administered intravenously, and serum TAG level was measured sequentially 0 (before injection), 1,2 , and $4 \mathrm{~h}$ after the reagent injection (e). The increasing rate of serum TAG level, which represents a TAG secretion rate from the liver, was calculated based on the regression line (f). [means \pm S.E.M., $(\mathbf{a}, \mathbf{c}, \mathbf{d}: \mathrm{n}=5 ; \mathbf{b}: \mathrm{n}=4 ; \mathbf{e}, \mathbf{f}: \mathrm{n}=6$; Student's t-test for $\mathbf{a}, \mathbf{b}$; Tukey-Kramer test for $\mathbf{c}, \mathbf{d}, \mathbf{f}$ )]. 
(a)
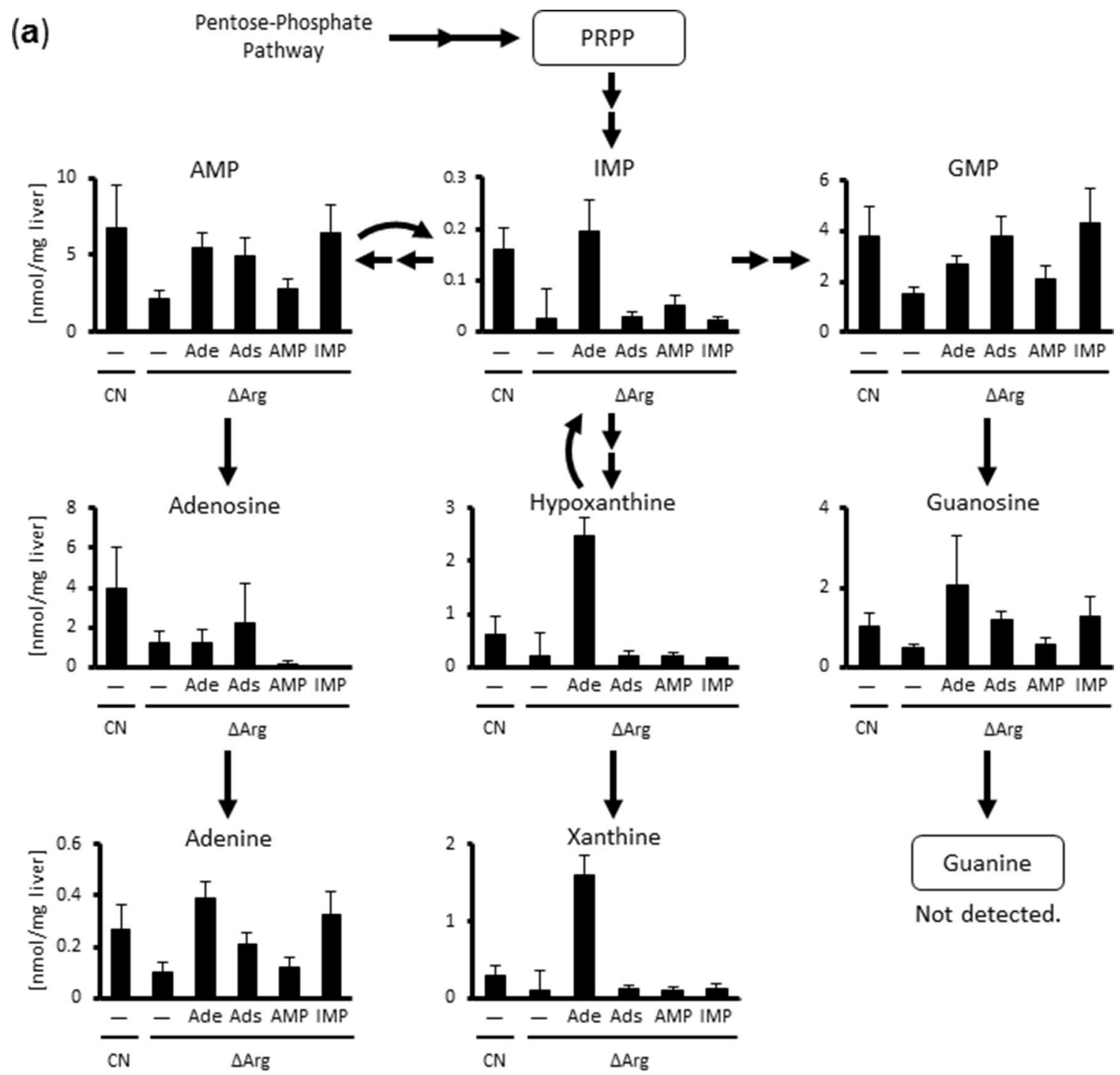

Not detected.

(b)

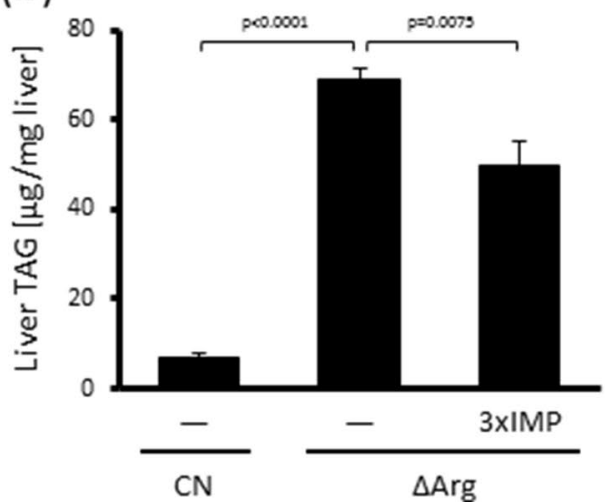

(c)

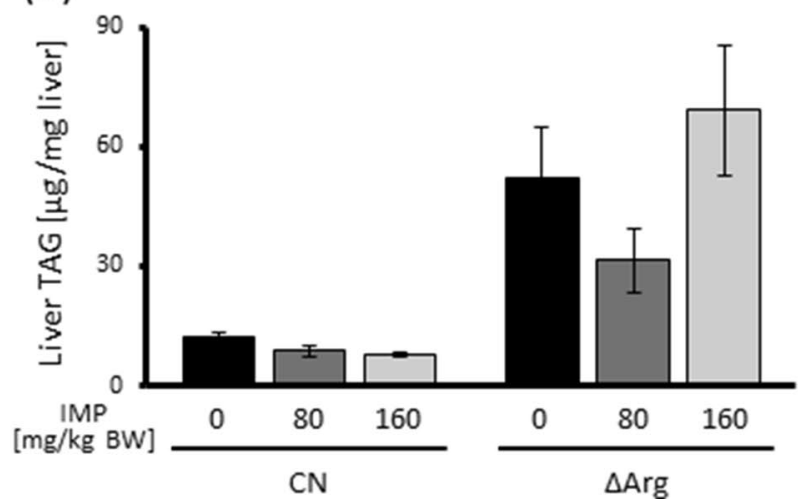

Figure 2. Adenine and related metabolites per se are unlikely to be involved in the hepatic lipid metabolic regulation. (a) Schematic diagram of purine metabolism and the levels of each metabolite in the livers of rats shown in Fig. 1d. (b) Six-week-old male Wistar rats were fed the CN diet or $\Delta$ Arg diet with or without $9 \mathrm{~g} / \mathrm{kg}$ IMP supplement for seven days. TAG levels in their livers were measured. (c) Six-week-old male Wistar rats were fed the CN diet or $\triangle \mathrm{Arg}$ diet for seven days. 80 or $160 \mathrm{mg} / \mathrm{kg}$ body wt. of IMP solution or just saline was injected via the tail vein at day 1,3 , and 5 after beginning of experimental diets. TAG levels in the livers were measured. 
profiles of rats fed the purine-supplemented diets in order to predict their hepatic TAG accumulation levels by using this method.

Figure 3a illustrates the program output of TAG values ("Predicted") based only on the serum amino acid data and comparison with the measured hepatic TAG levels ("True"). Remarkably, the inhibitory effect of adenine supplementation on TAG accumulation was estimated with high accuracy, suggesting that adenine supplementation to the $\triangle \mathrm{Arg}$ diet would probably change the serum amino acid profile. Thus, we compared the serum amino acid profiles of each rat ( $\triangle \mathrm{Arg}, \Delta \mathrm{Arg}+$ adenine, $\Delta \mathrm{Arg}+$ adenosine, $\Delta \mathrm{Arg}+\mathrm{AMP}$, and $\Delta \mathrm{Arg}+\mathrm{IMP})$, and found that the serum amino acid profiles of the "fatty liver group" ( $\triangle \mathrm{Arg}, \Delta \mathrm{Arg}+$ adenosine, $\Delta \mathrm{Arg}+\mathrm{AMP}, \Delta \mathrm{Arg}+\mathrm{IMP})$ were quite similar to each other, but that of the $\Delta \mathrm{Arg}+$ adenine group greatly differed (Fig. $3 \mathrm{~b}$, Supplementary Table S4). These data suggested that adenine supplementation to a $\Delta$ Arg diet, but not that of the other metabolites, changed the serum amino acid profile towards a "non-inducible pattern" for hepatic lipid accumulation.

Alterations in the serum amino acid profile prevent hepatic TAG accumulation caused by a $\Delta$ Arg diet. Finally, to confirm the causative effect of serum amino acid profiles on hepatic TAG accumulation in vivo, we conducted different dietary interventions with the aim of perturbating serum amino acid compositions. Comparing the serum amino acid concentrations of rats fed a $\Delta \operatorname{Arg}$ diet and a $\Delta \operatorname{Arg}+$ adenine diet, the levels of methionine, histidine, and branched-chain amino acids ([BCAAs]; valine, leucine, isoleucine) were found to be much lower in $\Delta A r g$ + adenine-fed rats (Fig. 3b,c). Subsequently, the concentration of each of these amino acids was reduced by two thirds in the $\Delta$ Arg diet and rats were fed with this new diet composition for seven days. As expected, the deficiency of methionine or BCAAs in the $\triangle$ Arg diet dramatically changed serum amino acid profiles and significantly abolished hepatic TAG accumulation, whereas histidine deficiency had little impact (Fig. 4, Supplementary Table S3, 4). Taken together, our results suggested that the specific serum amino acid profile formed in response to a $\triangle \mathrm{Arg}$ diet was able to regulate the lipid metabolism in hepatocytes, where attenuated TAG secretion caused TAG accumulation, leading to the development of fatty liver.

\section{Discussion}

Dietary imbalance of some nutrients, including proteins/amino acids and nitrogenous bases, has been reported to have a significant impact on neutral lipid accumulation in the liver ${ }^{3,6}$. The amino acids, namely arginine, methionine, threonine, tryptophan, and glutamine has been suggested in NAFL ${ }^{3,5,18}$, but the underlying molecular mechanisms for this phenomenon have been described in a highly controversial manner with contradictory results. For example, while a methionine/choline-deficient diet is well known to induce NAFLD, a methioninedeficient diet prevents fatty liver progression in $o b / o b$ mice $^{19,20}$. Furthermore, a low-arginine or low-threonine diet induced fatty liver development in rats, whereas arginine or threonine supplementation to an amino aciddeficient diet failed to reverse fatty liver development ${ }^{5}$. These results strongly suggest that monitoring the intake of a single amino acid is not sufficient to understand the whole scenario of NAFL progression associated with amino acid nutrition. In this study, we provide another perspective for this relationship: the serum amino acid profile.

Adenine reportedly has an inhibitory effect on NAFL progression induced by a low-arginine diet, although the underlying mechanism is still unknown ${ }^{6}$. Some metabolites in purine metabolism, such as uric acid, are known as antioxidants; thus, it is possible that antioxidative properties of these molecules may have hepatoprotective effects $^{21}$. If adenine itself is a bioactive molecule for controlling hepatic lipid metabolism, the ingested adenine should reach the liver through blood circulation. In addition, other purine metabolites should also change hepatic TAG levels because they could affect the adenine concentration through nucleotide metabolism. However, in the present study, even when adenine was supplemented in the diet, serum adenine concentrations were very low, often below the detectable level. Moreover, the hepatic adenine content did not correlate with hepatic TAG levels, although IMP actually increased adenine concentration in the liver (Fig. 2a). Since we previously discovered that dietary amino acid composition can dramatically affect serum amino acid profiles, changing it into an unexpected pattern ${ }^{5}$, we hypothesized that dietary adenine intake would also modify the serum amino acid profiles, which in turn would lead to a down-regulation of hepatic TAG accumulation. Indeed, oral adenine administration, but not other metabolites, modified serum amino acid profiles, and a medium amino acid composition that was similar to the serum of $\triangle$ Arg diet-fed rats enhanced TAG accumulation in a cultured hepatocyte model (Fig. 1a,b). The media used in this study were serum-free, indicating that the amino acid profile was a sufficient signal for cellular metabolic regulation, regardless of any hormonal stimulation or neuronal input. These results illustrate a novel type of "metabolic regulatory amino acid signal" driven by comprehensive serum amino acid profiles.

In the current study, we used data of 20 protein-composing amino acids plus cystine for machine learning analyses, but it was not clear whether all these amino acids were indeed necessary for signal transduction. Although the $\Delta \mathrm{Arg}$ diet increased both methionine and histidine levels in the serum, a $\Delta \mathrm{Arg} / \Delta \mathrm{His}$ diet did not exhibit an apparent effect on hepatic TAG levels, whereas a $\Delta \mathrm{Arg} / \Delta$ Met diet significantly lowered these levels (Figs. 3b,c, 4). These results suggest that not all amino acids are essential to determine hepatic TAG levels. In addition, serum BCAA levels were not increased by a $\triangle A$ rg diet, but were decreased by adenine supplementation, and hepatic TAG accumulation was inhibited by a $\triangle$ Arg/ $\triangle$ BCAA diet (Fig. $2 b, 3 b$ ), implying that not only the absolute amino acid concentrations but also their relative concentrations are important. Furthermore, it was shown that the activities of peroxisome proliferator-activated receptor $\alpha$ (PPAR $\alpha)$ and sterol regulatory element-binding protein-1c (SREBP1c), which are generally considered to be important transcription factors for the regulation of hepatic lipid metabolism, were not affected by amino acid deficiency ${ }^{5}$, suggesting that another mechanism is likely to be involved. Further research is necessary to understand which specific combinations of amino acids are actually functional and how these signals are recognized by the hepatocytes, leading to modifications in the activities of lipid metabolic machinery. 
Figure 3. Dietary adenine affects hepatic lipid metabolism through modification of serum amino acid profile. (a) TAG levels in the individual liver of rats shown in Fig. 1d was estimated based on the data of their serum amino acid concentrations by multilayer perceptron (MLP). See Method section and reference $\mathrm{f}^{5}$ for detail. Briefly, learning was conducted using serum amino acid concentrations of 95 rats as input data and putting corresponding measured liver TAG level in the output. All values used in this calculation were standardized and presented in the arbitrary unit. (b) Sera of rats shown in Fig. 1d were collected and their amino acid concentration was measured. Data are shown as a fold of the mean value of the CN group (dotted line), and average values of each experimental diet group (continuous line) are indicated. (c) The same data as (b), but shown as a fold of the mean value of the $\Delta$ Arg group (dotted line).

Altogether, we conclude that changes in comprehensive serum amino acid profiles in response to dietary amino acid compositions play a causative role in the regulation of lipid metabolism in the liver, and dietary adenine interferes with this signal transmission by perturbating serum amino acid profiles. This discovery should provide a novel insight into NAFLD pathophysiology. Although there are several exceptions, such as hepatitis $C$ virus-induced NAFLD, which is unlikely to have a direct link with serum amino acids ${ }^{22}$, a certain part of the mechanism of pathogenesis should be explained by the amino acid signals that we discovered. This will lead to the design of useful diagnostic methods and establishment of effective prevention or treatment for NAFLD.

\section{Materials and methods}

Materials. For animal experimental diets, the vitamin mixture, mineral mixture, cellulose powder, and corn starch were purchased from Oriental Yeast Co. (Tokyo, Japan), while soybean oil was acquired from Nacalai Tesque (Tokyo, Japan). Solutions such as $10 \times$ Earle's buffered salt solution (EBSS) and $100 \times$ MEM vitamin solution were purchased from Sigma-Aldrich (St. Louis, MO, USA). Dulbecco's modified Eagle's medium (DMEM) was purchased from Nissui Pharmaceutical Co. (Tokyo, Japan). Fetal bovine serum (FBS) was obtained from Sigma-Aldrich. Penicillin and streptomycin were obtained from Banyu Pharmaceutical Co., (Ibaraki, Japan). Other chemicals were of commercially available reagent grade.

Animal experiments. Five-week-old male Wistar rats were purchased from Charles River Japan (Kanagawa, Japan). The rats were caged individually and maintained at $24 \pm 1{ }^{\circ} \mathrm{C}$ with $50-60 \%$ humidity and a $12 \mathrm{~h}$ light/dark cycle (8:00-20:00/ 20:00-8:00). They were allowed free access to food and water during the experiment.

Prior to the experiments, all rats were fed normal chow for three days and a control (CN) diet containing $15 \%$ (w/w) amino acids (Supplementary Table S1) for the next four days as a prefeeding period. The animals were then divided into experimental groups. Thereafter, each group was given either the $\mathrm{CN}$ diet or each experimental diet for seven days. In a specific amino acid-deficient diet, the indicated amino acid concentration was $1 / 3$ of that of the $\mathrm{CN}$ diet, and the concentration of the nucleotide supplement was $3 \mathrm{~g} / \mathrm{kg}$ unless otherwise stated. For IMP injections, an inosine-5'-monophosphate disodium salt solution (100 mM, Sigma-Aldrich) was injected in the tail vein at days 1,3 , and 5 after beginning the experimental diets. In the control group, the same volume of saline solution was injected in the tail vein. All the diets were self-made. Throughout the experimental period, the body weight and food intake of all rats were measured at 10:00 AM every day. For tissue collection, rats were anesthetized with isoflurane (DS Pharma Animal Health, Tokyo, Japan) and then samples were obtained from the rats' carotid arteries and livers. The serum was prepared soon after blood collection and liver samples were immediately frozen in liquid nitrogen. All samples were stored at $-80^{\circ} \mathrm{C}$ for future use.

All animal care and experiments conformed to the Guidelines for Animal Experiments of The University of Tokyo and were approved by the Animal Research Committee of The University of Tokyo.

Cell culture and experiments. H4IIE-C3 cells (a rat hepatoma cell line, ATCC CRL-1600) were grown in DMEM supplemented with $10 \% \mathrm{FBS}$ and antibiotics under $5 \% \mathrm{CO}_{2}$ at $37^{\circ} \mathrm{C}$. Upon reaching sub-confluency, the medium was changed to an experimental serum-free medium (Supplementary Table S2), and the cells were further cultured for $24 \mathrm{~h}$.

Liver/cellular TAG measurement. Total lipids in the liver were extracted according to Folch's method ${ }^{23}$ with small modifications. The frozen liver was homogenized in methanol:chloroform solution $(1: 2, \mathrm{v} / \mathrm{v})$, followed by the addition of $20 \%$ volume of $0.8 \% \mathrm{KCl}$ solution and centrifugation at $13,000 \times \mathrm{g}$, at $4{ }^{\circ} \mathrm{C}$ for $10 \mathrm{~min}$. Then, the organic (chloroform) layer was collected, the solvent was evaporated, and the remaining lipids were reconstituted in isopropanol. Similarly, lipids in cultured cells were extracted with a methanol:chloroform solution $(1: 1, v / v)$ and a saturated $\mathrm{NaCl}$ solution. The TAG content in the lipid extract was measured using Triglyceride E-test Wako (Wako, Osaka, Japan).

MLP analysis. We built an MLP program using the libraries Keras, version 2.3.1, and TensorFlow, version 2.1.0, previously ${ }^{5}$, and we used the program in the present study with no modifications. The training data set was composed of serum amino acid profiles and liver TAG levels of each rat that were obtained in the same previous study. The MLP network configuration was as follows: a 21-dimensional input (20 amino acids + cystine), 4 hidden layers with 300, 300, 300, and 100 neurons from the input direction, and a 1-dimensional output of liver TAG levels. Dropouts were provided in each layer and set to $0.5,0.4,0.4,0.4$, and 0.3 from the input direction. The maximum learning epoch was set to 10,000 . If the loss values did not update after more than 1,000 training epochs, "early stopping" was used to stop the training. Mean square error was used for the Loss function, and 
(a)

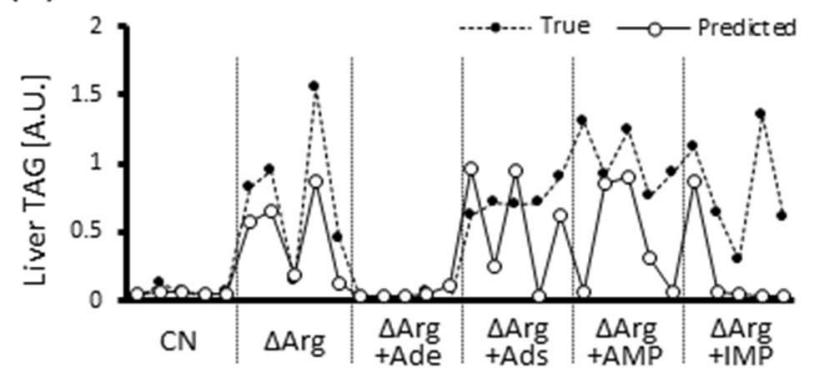

(b)
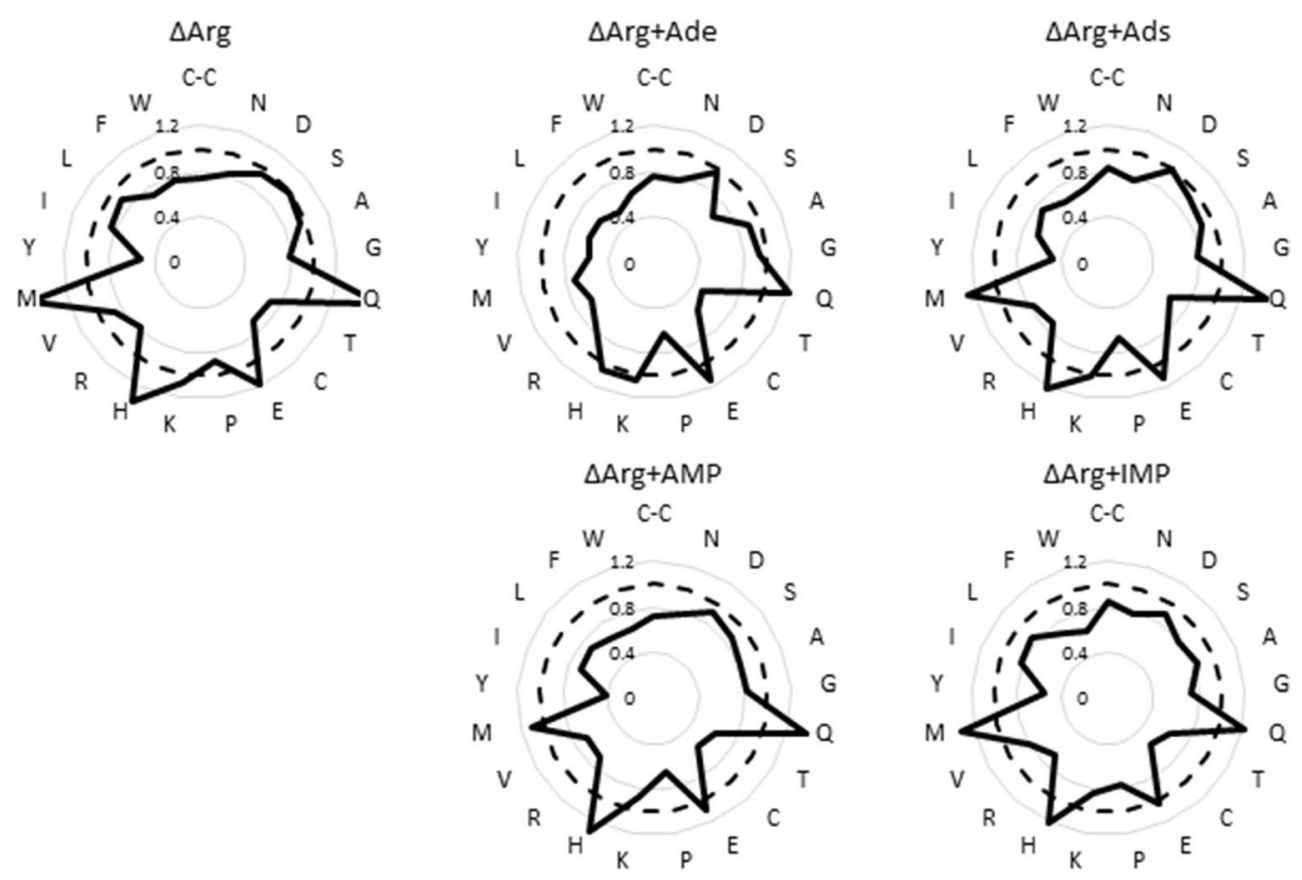

(c)
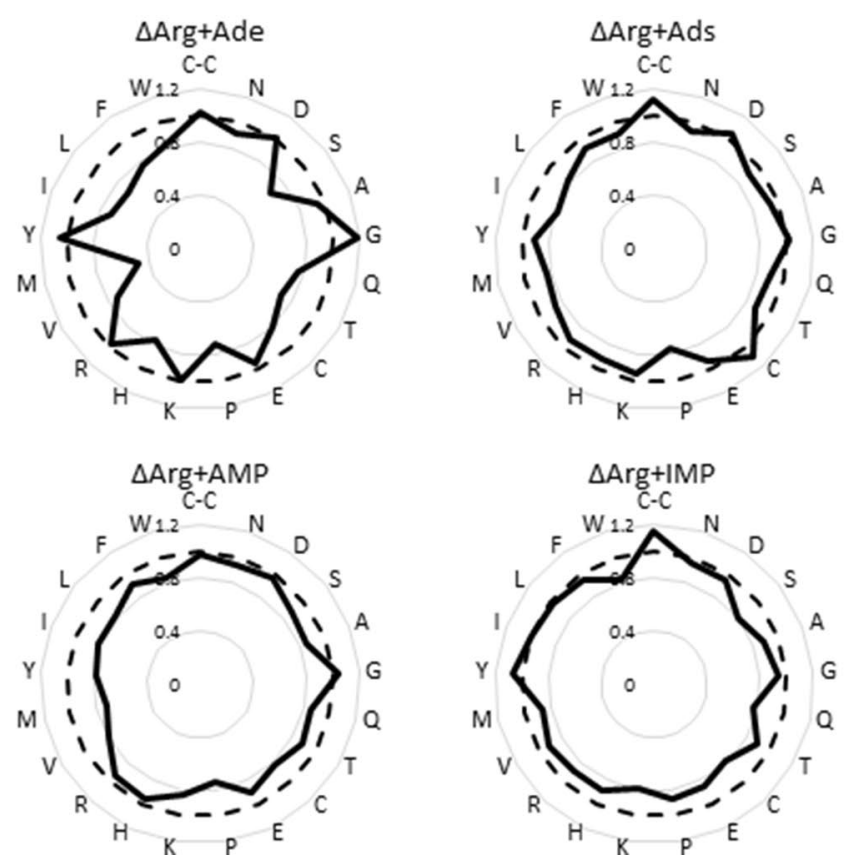
(a)

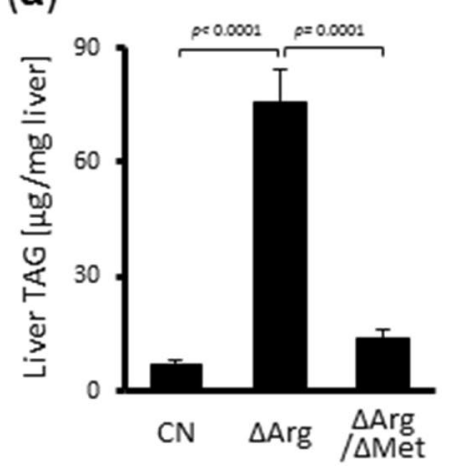

(d)

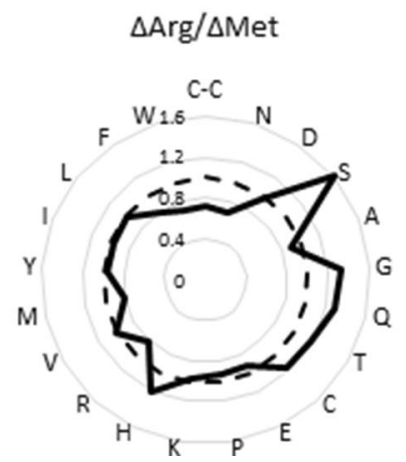

(b)
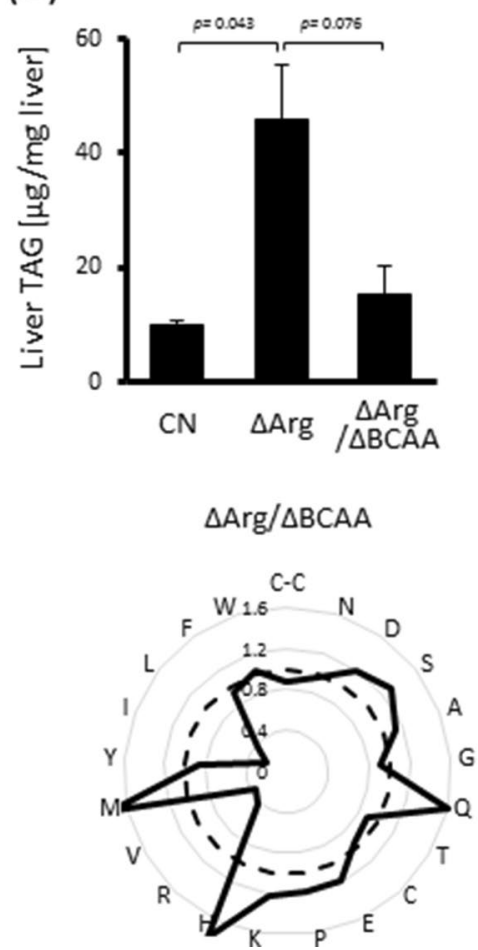

(c)
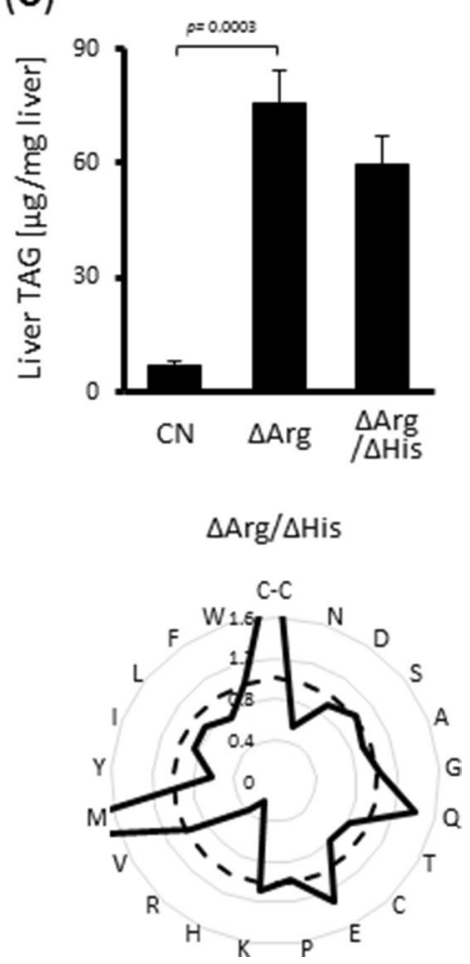

Figure 4. Perturbation of serum amino acid profile reverses arginine deficient diet-induced lipid accumulation in the liver. (a-c) Six-week-old male Wistar rats were fed the $\mathrm{CN}$ diet, $\Delta$ Arg diet, both arginine and methionine deficient diet $(\triangle \mathrm{Arg} / \Delta \mathrm{Met})(\mathbf{a})$, both arginine and BCAAs deficient diet $(\triangle \mathrm{Arg} / \triangle \mathrm{BCAA})(\mathbf{b})$, or both arginine and histidine deficient diet ( $\triangle \mathrm{Arg} / \Delta \mathrm{His})$ (c) for seven days. TAG levels in their livers. (d) Serum amino acid concentrations of rats shown in $(\mathbf{a}-\mathbf{c})$ were measured. Data of serum amino acid concentration are shown as a fold of the mean value of the $\mathrm{CN}$ group (dotted line), and average values of each experimental diet group (continuous line) are indicated. [bar; means \pm S.E.M., $(n=4)$, Tukey-Kramer test].

RMSprop for the optimization function. The activation function used ReLU in each layer. The trained model was used to predict liver TAG levels from untrained data sets of serum amino acid concentrations.

Serum and liver metabolite analysis. For serum metabolite extraction, $50 \mu \mathrm{L}$ of serum samples were mixed on ice with $120 \mu \mathrm{L}$ methanol containing internal control substances: $25 \mu \mathrm{M} 2$-morpholinoethanesulfonic acid and $100 \mu \mathrm{M}$ methionine sulfone. After centrifugation $\left(16,000 \times g\right.$ for $10 \mathrm{~min}$ at $\left.4{ }^{\circ} \mathrm{C}\right), 130 \mu \mathrm{L}$ of supernatant was mixed with $250 \mu \mathrm{L}$ ultrapure water and subjected to ultrafiltration using $3 \mathrm{kDa}$ cutoff filters (Amicon Ultra $3 \mathrm{~K}$ device, Merck, Darmstadt, Germany), followed by 30 min of evaporation and $6 \mathrm{~h}$ of lyophilization.

For liver metabolite extraction, approximately $100 \mathrm{mg}$ of liver pieces were homogenized in $500 \mu \mathrm{L}$ of methanol containing internal controls. The homogenates were diluted with $250 \mu \mathrm{L}$ of ultrapure water, and $600 \mu \mathrm{L}$ of each homogenate was mixed with $400 \mu \mathrm{L}$ chloroform. Following centrifugation $\left(16,000 \times g\right.$ for 5 min at $\left.4{ }^{\circ} \mathrm{C}\right), 800 \mu \mathrm{L}$ of the upper phase was collected and subjected to $30 \mathrm{~min}$ of evaporation. Then, $300 \mu \mathrm{L}$ of ultrapure water was added, followed by ultrafiltration and lyophilization.

The lyophilized metabolite samples were reconstituted in $200 \mu \mathrm{L}$ ultrapure water, further diluted if necessary, and then subjected to LC-MS/MS (LCMS-8030, Shimadzu, Kyoto, Japan) analysis, using Method Package for Primary Metabolites ver. 2 (Shimadzu) according to the manufacturer's protocol.

TAG secretion assay. Six-week-old male Wistar rats were fed $\mathrm{CN}$ or $\Delta$ Arg diets with or without nitrogenous base supplementation for seven days. Then, $200 \mathrm{mg} / \mathrm{kg}$ body weight of tyloxapol dissolved in $0.9 \% \mathrm{NaCl}$ (Sigma-Aldrich) was administered into the tail vein $(200 \mathrm{mg} / \mathrm{kg}$ body weight) under isoflurane anesthesia (DS Pharma Animal Health) and blood was collected 0, 1, 2, and $4 \mathrm{~h}$ after injection. TAG concentrations in the sera were measured using Triglyceride E-test Wako (Wako). Because tyloxapol is an inhibitor of endogenous lipoprotein lipase, TAG accumulation in the blood after its injection under fasting conditions reflects TAG secretion from the liver. Thus, the TAG increasing rate in the serum after tyloxapol injection was interpreted as the TAG secretion activity in the liver.

Statistical analysis. Data are expressed as mean \pm standard error of the mean (SEM), unless otherwise stated. Comparisons between two groups were performed using Student's $t$-test. Comparisons among more than two groups were performed using one-way analysis of variance (ANOVA). If the $p$ value obtained from the 
ANOVA test was $<0.05$, the Tukey-Kramer post-hoc test was performed. Values of $p<0.05$ were considered statistically significant. All statistical calculations were performed using JMP Pro (SAS Institute Inc., Cary, NC, USA).

\section{Data availability}

All data shown in this manuscript are available from the corresponding author on reasonable request.

Received: 28 September 2020; Accepted: 4 December 2020

Published online: 17 December 2020

\section{References}

1. Spengler, E. K. \& Loomba, R. Recommendations for diagnosis, referral for liver biopsy, and treatment of nonalcoholic fatty liver disease and nonalcoholic steatohepatitis. Mayo Clin. Proc. 90, 1233-1246 (2015).

2. Zivkovic, A. M., German, J. B. \& Sanyal, A. J. Comparative review of diets for the metabolic syndrome: implications for nonalcoholic fatty liver disease. Am. J. Clin. Nutr. 86, 285-300 (2007).

3. Hernandez-Rodas, M., Valenzuela, R. \& Videla, L. Relevant aspects of nutritional and dietary interventions in non-alcoholic fatty liver disease. Int. J. Mol. Sci. 16, 25168-25198 (2015).

4. Toyoshima, Y., Tokita, R., Taguchi, Y., Akiyama-akanishi, N. \& Takenaka, A. Tissue-specific effects of protein malnutrition on insulin signaling pathway and lipid accumulation in growing rats. Endocr. J. 61, 499-512 (2014).

5. Nishi, H. et al. Importance of serum amino acid profile for induction of hepatic steatosis under protein malnutrition. Sci. Rep. 8, $1-11(2018)$.

6. Aoyama, Y., Yoshida, A. \& Ashida, K. Effect of some dietary additions to either an arginine- devoid diet or a diet supplemented with orotic acid refed after starvation on liver lipid content during essential fatty acid deficiency in rats. J. Nutr. 111, 895-906 (1981).

7. Marchetti, M., Puddu, P. \& Caldarera, C. Metabolic aspects of 'orotic acid fatty liver'. Nuleotide control mechanisms of lipid metabolism. Biochem. J. 92, 46-51 (1964).

8. Hassan, A. S. \& Milner, J. A. Alterations in liver nucleic acids and nucleotides in arginine deficient rats. Metabolism 30, 739-744 (1981).

9. Milner, J. A. Metabolic aberrations associated with arginine deficiency. J. Nutr. 115, 516-523 (1985).

10. Milner, J. A. Mechanism for fatty liver induction in rats fed arginine deficient diets. J. Nutr. 109, 24-31 (1979).

11. Angulo, P. Nonalcoholic fatty liver disease. N. Engl. J. Med. 346, 1221-1231 (2002).

12. Wong, V.W.-S., Adams, L. A., de Lédinghen, V., Wong, G.L.-H. \& Sookoian, S. Noninvasive biomarkers in NAFLD and NASHcurrent progress and future promise. Nat. Rev. Gastroenterol. Hepatol. 15, 461-478 (2018).

13. Otani, L. et al. Low-arginine and low-protein diets induce hepatic lipid accumulation through different mechanisms in growing rats. Nutr. Metab. (Lond) 17, 1-13 (2020).

14. Noguchi, Y. et al. Network analysis of plasma and tissue amino acids and the generation of an amino index for potential diagnostic use. Am. J. Clin. Nutr. 83, 513-519 (2006).

15. Gaggini, M. et al. Altered amino acid concentrations in NAFLD: impact of obesity and insulin resistance. Hepatology 67, 145-158 (2018).

16. Lynch, C. J. \& Adams, S. H. Branched-chain amino acids in metabolic signalling and insulin resistance. Nat. Rev. Endocrinol. 10, 723-736 (2014).

17. Miyagi, Y. et al. Plasma free amino acid profiling of five types of cancer patients and its application for early detection. PLoS ONE 6, e24143 (2011).

18. Aoyama, Y., Yasui, H. \& Ashida, K. Effect of dietary protein and amino acids in a choline-deficient diet on lipid accumulation in rat liver. J. Nutr. 101, 739-745 (1971).

19. Itagaki, H., Shimizu, K., Morikawa, S., Ogawa, K. \& Ezaki, T. Morphological and functional characterization of non-alcoholic fatty liver disease induced by a methionine-choline-deficient diet in C57BL/6 mice. Int. J. Clin. Exp. Pathol. 6, 2683-2696 (2013).

20. Malloy, V. L. et al. Methionine restriction prevents the progression of hepatic steatosis in leptin-deficient obese mice. Metabolism 62, 1651-1661 (2013).

21. Ames, B. N., Cathcart, R., Schwiers, E. \& Hochstein, P. Uric acid provides an antioxidant defense in humans against oxidant- and radical-caused aging and cancer: a hypothesis. Proc. Natl. Acad. Sci. U. S. A. 78, 6858-6862 (1981).

22. Patel, A. \& Harrison, S. A. Hepatitis C virus infection and nonalcoholic steatohepatitis. Gastroenterol. Hepatol. 8, 305-312 (2012).

23. Folch, J. \& Lees, M. S. G. A simple method for the isolation and purification of total lipides from animal tissues. J. Biol. Chem. 55, 999-1033 (1957).

\section{Acknowledgments}

We thank the members of Professor Takahashi's laboratory for their valuable support and discussion and Ms. Tomomi Ueda and Ms. Noyumi Nakamura of Dr. Ito's laboratory for their technical support. We also appreciate Drs. Susan Hall and Narula Acharan Singh (University of North Carolina at Chapel Hill) for imparting scientific suggestions to improve the manuscript. Finally, we would like to thank Editage (www.editage.com) for their English language editing services.

\section{Author contributions}

H.N, D.Y., M.M., Y.G., F.H., and S-I. T. designed the experiments. H.N. and D.Y. conducted the wet lab experiments and M.M. performed machine learning analysis. K.I., F.H., and S-I. T critically supervised the study. H.N, D.Y., M.M., F.H., and S-I. T. wrote this manuscript.

\section{Funding}

This work was supported in part by Grants-in-Aid for Scientific Research (B) \#15H04583 and Scientific Research (B) \#24380152 from the Japan Society for the Promotion of Science (JSPS) to F.H.; Grant-in-Aid for the Scientific Research (S) \#25221204 and Scientific Research (A)\#18H03972, as well as Core-to-core program A. A. Advanced Research Networks from JSPS and R\&D matching funds on the Field for Knowledge Integration and Innovation from the Bio-oriented Technology Research Advancement Institution (NARO), to S-I.T. Grant-in-Aid for the Scientific Research (C) \#19K06436 from JSPS to D.Y.; Collaborative research with For Days Co., Ltd. to K.I. 


\section{Competing interests}

The authors declare no competing interests.

\section{Additional information}

Supplementary Information The online version contains supplementary material available at https://doi. org/10.1038/s41598-020-79234-w.

Correspondence and requests for materials should be addressed to F.H.

Reprints and permissions information is available at www.nature.com/reprints.

Publisher's note Springer Nature remains neutral with regard to jurisdictional claims in published maps and institutional affiliations.

(c) (1) Open Access This article is licensed under a Creative Commons Attribution 4.0 International License, which permits use, sharing, adaptation, distribution and reproduction in any medium or format, as long as you give appropriate credit to the original author(s) and the source, provide a link to the Creative Commons licence, and indicate if changes were made. The images or other third party material in this article are included in the article's Creative Commons licence, unless indicated otherwise in a credit line to the material. If material is not included in the article's Creative Commons licence and your intended use is not permitted by statutory regulation or exceeds the permitted use, you will need to obtain permission directly from the copyright holder. To view a copy of this licence, visit http://creativecommons.org/licenses/by/4.0/.

(C) The Author(s) 2020 